\title{
Computing Dynamic Output Feedback Laws
}

\author{
Jan Verschelde* $\quad$ Yusong Wang ${ }^{\dagger}$
}

2 June 2003

\begin{abstract}
The pole placement problem asks to find laws to feed the output of a plant governed by a linear system of differential equations back to the input of the plant so that the resulting closed-loop system has a desired set of eigenvalues. Converting this problem into a question of enumerative geometry, efficient numerical homotopy algorithms to solve this problem for general Multi-Input-Multi-Output (MIMO) systems have been proposed recently. While dynamic feedback laws offer a wider range of use, the realization of the output of the numerical homotopies as a machine to control the plant in the time domain has not been addressed before. In this paper we present symbolic-numeric algorithms to turn the solution to the question of enumerative geometry into a useful control feedback machine. We report on numerical experiments with our publicly available software and illustrate its application on various control problems from the literature.

2000 Mathematics Subject Classification. Primary 93B55. Secondary 14Q99, 65H10, 68W30, 93B27.

Key words and phrases. Control of linear systems, dynamic output feedback, Multi-Input-Multi-Output (MIMO) systems, numerical homotopy algorithms, numerical Schubert calculus, pole placement, symbolicnumeric computations.
\end{abstract}

\section{Introduction}

Given a linear system of differential equations and a list of eigenvalues, the pole placement problem asks to find laws to feed the output back to the input so that the resulting closed-loop system has the same eigenvalues as the given list.

${ }^{*}$ Department of Mathematics, Statistics, and Computer Science, University of Illinois at Chicago, 851 South Morgan (M/C 249), Chicago, IL 60607-7045, USA. Email: jan@math.uic.edu or jan.verschelde@na-net.ornl.gov. URL: http://www.math.uic.edu/ jan This material is based upon work supported by the National Science Foundation under Grant No. 0105739 and Grant No. 0134611.

${ }^{\dagger}$ Department of Mathematics, Statistics, and Computer Science, University of Illinois at Chicago, 851 South Morgan (M/C 249), Chicago, IL 60607-7045, USA. Email: ywang25@uic.edu. 
While executing a static feedback law corresponds to a simple matrix-vector multiplication, a dynamic compensator has several internal states.

The theoretical solution of this problem was found in the Schubert calculus, see [3, 4] for the static, and [25, 26] for dynamic compensators. Because of its importance to practical applications, the development of algorithms for this problem was stated as an open problem 28 (see also [5]). The first homotopy algorithms were proposed in [14], defining a numerical Schubert calculus. The Pieri homotopy algorithms of [14 were improved and generalized to dynamic feedback in [15]. In [20], the numerical performance of these homotopies for static feedback was improved.

Our current paper is a sequel to our previous paper [35] as a continuing effort to apply the homotopy algorithms to practical applications of linear systems control. Rather than generating random matrices as inputs to our algorithms we look to build case studies with examples from the literature. In 35] we applied static output feedback to use pole placement to keep a satellite in orbit. In this current paper we consider the application of dynamic feedback laws to this and other applications. See [18] for the relation of the pole placement problem with inverse eigenvalue and matrix extension problem.

As expected, with the more general dynamic feedback laws we can cover a wider range of applications, but at the same time we experienced the real need for more advanced algorithms from numerical algebraic geometry to deal better with over and underdetermined problems. We define these mathematical problems in the next section.

The heart of this paper concerns the realization of dynamic compensators. As these compensators are computed in the frequency domain, at the end of the paths defined by the homotopies, any application demands the description of the controllers in the time domain as a tuple of matrices. While realization algorithms are covered in standard graduate textbooks (e.g., [1] and [16]), our output is numerical, i.e.: subject to roundoff and known only with limited accuracy. Reports on numerical implementations of algorithms which manipulate polynomial matrices are scarce, we found only one paper [12], which presents a numerical study on a commercial implementation, but leaves the implementation of a numerical Smith normal form as an open question. Note that the Smith normal form plays an important role to obtain a minimal realization of the transfer function of Multi-Input-Multi-Output (MIMO) systems.

Section three starts with the description of an algorithm to compute the greatest common divisor of two polynomials with approximate coefficients, as this is the cornerstone function in the implementation of a numerical Smith normal form. As we experienced and illustrate, the naive application of the Euclidean algorithm for an approximate GCD can fail miserably. That many algorithms to solve problems with exact data turn out to be numerically unstable is a growing concern in computer algebra, which has led to hybrid symbolicnumeric computation [6]. In particular, the approximate GCD problem has received a lot of research attention in recent years, see for instance [2] 8], 13], 
[17, 24], 37] and 38.

Our approach is to consider the input polynomials not as polynomials with approximate coefficients, but as polynomials defined by approximate roots. So we view polynomials not symbolically (as sums of monomials with approximate coefficients) but geometrically (as the collection of roots in the complex plane, known with limited accuracy). The idea to computing the GCD by matching common approximate roots (within a certain tolerance) can be found in 24. Compared (see 24]) to alternative constructions of approximate GCDs, this method works well for input polynomial with multiple or clustered zeros. Algorithmically, we can immediately apply any numerical root finding method (see 23] for a recent survey). To compute the polynomials in the extended GCD problem (i.e.: the $k(x)$ and $l(x)$ in the combination $k(x) a(x)+l(x) b(x)=\operatorname{GCD}(a(x), b(x)))$, we apply Newton interpolation.

As usual, the link between algorithms and applications is software. We describe an extension to the software package PHCpack [34] in section five before illustrating its application on various control problems from the literature.

\section{Problem Statement}

The algorithmic framework is defined by the transitions between the time and the frequency domain (which leads to the symbolic-numeric calculations). Mathematically, we count the number of conditions to determine the dimension of our solution space.

In the time domain, we picture the control of a plant with a dynamic feedback

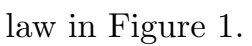

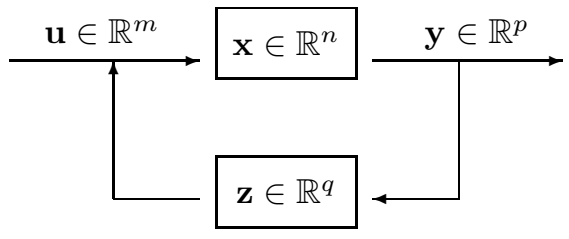

Figure 1: Control of an $m$-input and $p$-output plant by a $q$ th order dynamic compensator in the time domain.

The machine we want to control is given by three matrices $(A, B, C)$, defining a system of first-order differential equations in the time domain. The input of the Pieri homotopies is sampled in the frequency domain. So to apply these homotopies, we need to translate the input from the time to frequency domain and to realize the output as a tuple of matrices. Table1defines the three stages in the data flow. The labels on the arrows in Figure 2 correspond to the numbers in Table 1 
(1) With plain linear algebra we compute the input for the Pieri homotopies, sampling points from the plant we wish to control.

(2) Pieri homotopies compute solution maps of degree $q$, which are then brought into another format.

(3) Given the transfer function of the compensator, we realize the compensator by a tuple of four matrices $(F, G, H, K)$.

Table 1: Transitions in applying the Pieri homotopies.

Our problem is thus to process the output of the Pieri homotopy algorithms and to apply realization algorithms to the numerical output.

In [15], the following equivalence relation was derived:

$$
\begin{aligned}
& \operatorname{det}\left(s\left[\begin{array}{cc}
I_{n} & 0 \\
0 & I_{q}
\end{array}\right]-\left[\begin{array}{cc}
A+B K C & B H \\
G C & F
\end{array}\right]\right)=0 \\
& \Leftrightarrow \operatorname{det}\left[\begin{array}{cc}
H\left(s I_{q}-F\right)^{-1} G+K & C\left(s I_{n}-A\right)^{-1} B \\
I_{m}
\end{array}\right]=0
\end{aligned}
$$

Equation (11) is the characteristic equation of the closed-loop system. Via elementary row and column operations, this equation can be rewritten into (2), which separates the given data $(A, B, C)$ from the unknown $(F, G, H, K)$. Equation (2) shows the geometric problem: we are looking for curves which produce $p$-planes in $\mathbb{C}^{m+p}$ which meet given $m$-planes sampled at prescribed values for $s$. In Figure 2 we show the transition between the time and the frequency domain.

$$
\begin{aligned}
& \left\{\begin{array} { l } 
{ \dot { \mathbf { x } } = A \mathbf { x } + B \mathbf { u } } \\
{ \mathbf { y } = C \mathbf { x } }
\end{array} \quad \left\{\begin{array}{l}
\dot{\mathbf{z}}=F \mathbf{z}+G \mathbf{y} \\
\mathbf{u}=H \mathbf{z}+K \mathbf{y}
\end{array}\right.\right. \\
& \text { (1) } \quad \uparrow(3) \\
& {\left[\begin{array}{c}
C\left(s I_{n}-A\right)^{-1} B \\
I_{m}
\end{array}\right] \stackrel{(2)}{\longrightarrow}\left[\begin{array}{c}
I_{p} \\
H\left(s I_{q}-F\right)^{-1} G+K
\end{array}\right]}
\end{aligned}
$$

Figure 2: Transitions between time and frequency domain, as defined in Table 1

When controlling a machine with $n$ internal states with a controller using $q$ internal states, we can place $n+q$ poles. On the other hand, the dimension of the geometric problem is $m p+q(m+p)$. Depending on values for $n, m, p$, and $q$, we distinguish three cases:

1) $n+q<m p+q(m+p)$ underdetermined: For a generic machine, there is a set of feedback laws. The set has dimension $m p+q(m+p)-n-q$, and 
has degree $d(m, p, q)$, i.e: for a generic choice of the parameters, we have $d(m, p, q)$ complex feedback laws. This degree can easily be computed combinatorially.

2) $n+q=m p+q(m+p)$ dimension zero: For a generic machine, there are exactly $d(m, p, q)$ complex feedback laws. Every feedback law places all $n+q$ poles at the desired locations. It may be that no feedback law has all its coefficients real, see [9, 10].

3) $n+q>m p+q(m+p)$ overdetermined: For a generic machine, there are no feedback laws which place all $n+q$ poles at the desired locations.

The numbers $m, p, n$ are fixed, given on input. We can choose $q$ to arrive always in a favorable condition and get feedback laws. In particular, if a system is overdetermined for static output feedback $(n>m p)$, we can always find $q$ to make it convert to underdetermined or dimension zero case with dynamic feedback. This is because $q(m+p)$ always increases faster than $q$.

Presently, we resolve the undetermined case by choosing additional input planes to the geometric problem. For nongeneric machines, the dimension of the set of feedback laws is higher than expected. Recent advances with homotopies (see e.g. [29, 30, 31, 32]) allow to treat positive dimensional solution sets, but we defer the application of these recent homotopy methods to a future paper.

\section{Symbolic-Numeric Calculations}

To transform a solution obtained by the homotopy methods into a description of a machine to execute the dynamic feedback law, we need to calculate the Smith normal form to compute the inverse of a matrix with polynomial entries. More precisely, the output of the homotopies as an $(m+p)$-by- $p$ matrix of polynomials in $s$ :

$$
\left[\begin{array}{l}
U(s) \\
V(s)
\end{array}\right]
$$

where $U(s)$ is a $p$-by- $p$ matrix and $V(s)$ is an $m$-by- $p$ matrix of polynomials in $s$, satisfies

$$
\operatorname{det}\left[\begin{array}{cc}
U(s) & C\left(s I_{n}-A\right)^{-1} B \\
V(s) & I_{m}
\end{array}\right]=0,
$$

for the given poles. We can right multiply (4) by

$$
\left[\begin{array}{cc}
U^{-1}(s) & 0 \\
0 & I_{m}
\end{array}\right]
$$

The result of this multiplication is

$$
\operatorname{det}\left[\begin{array}{cc}
I_{p} & C\left(s I_{n}-A\right)^{-1} B \\
V(s) U^{-1}(s) & I_{m}
\end{array}\right]=0 .
$$


Since the multiplier matrix (5) is of full rank, its determinant is nonzero and the original intersection condition remains. This multiplication does not affect the input conditions, which are at the right part of (2). By comparing (21) with (6), we can apply the realization algorithms to extract $(F, G, H, K)$ from the matrix $V(s) U^{-1}(s)$.

In the next subsection we show how the calculation of a Smith normal form requires the calculation of greatest common divisor.

\subsection{Numerical Smith Normal Form}

The Smith normal form of a matrix of polynomials can be seen as the discrete analogue of the singular value decomposition. More precisely, for any $n$-by$m$ matrix $A(x)$ whose entries are polynomials in $x$, there exists a unimodular $n$-by- $n$ matrix $P(x)$ and a unimodular $m$-by- $m$ matrix $Q(x)$ so that

$$
P(x) A(x) Q(x)=D(x),
$$

where $D(x)$ is an $n$-by- $m$ matrix which has only nonzero polynomials in $x$ on its diagonal. Furthermore, denoting the $i$ th element on the diagonal of $D(x)$ by $D_{i}$, we have that $D_{i}$ divides $D_{i+1}$. The matrix $D(x)$ is called the Smith normal form of $A(x)$. Since unimodular matrices are invertible, we can rewrite $A(x)$ as

$$
A(x)=P^{-1}(x) D(x) Q^{-1}(x),
$$

which reveals the following expression for the inverse of $A(x)$ :

$$
A^{-1}(x)=Q(x) D^{-1}(x) P(x),
$$

which of course only exists if $D(x)$ has full rank. We get the inverse $D^{-1}(x)$ by inverting every entry on the diagonal of $D(x)$.

The Smith normal form can be computed by solving the extended greatest common divisor problem. In particular, we wish to find polynomials $k(x)$ and $l(x)$ so that the following equality holds:

$$
d(x)=\operatorname{GCD}(a(x), b(x))=k(x) a(x)+l(x) b(x) .
$$

The calculation of the greatest common divisor is used to reduce columns

$$
\left[\begin{array}{rr}
k(x) & l(x) \\
-\frac{b(x)}{d(x)} & \frac{a(x)}{d(x)}
\end{array}\right]\left[\begin{array}{c}
a(x) \\
b(x)
\end{array}\right]=\left[\begin{array}{c}
d(x) \\
0
\end{array}\right]
$$

or to reduce rows

$$
\left[\begin{array}{ll}
a(x) & b(x)
\end{array}\right]\left[\begin{array}{rr}
k(x) & -\frac{b(x)}{d(x)} \\
l(x) & \frac{a(x)}{d(x)}
\end{array}\right]=\left[\begin{array}{ll}
d(x) & 0
\end{array}\right] .
$$

The matrices we use in the column and row reductions have determinant equal to one, they are called unimodular.

Collecting the column reductions in $P(x)$ and the row reductions in $Q(x)$, we can reduce any polynomial matrix $A(x)$ to a diagonal form $D(x)$, and so obtain the Smith normal form of $A(x)$. 


\subsection{Numerical Greatest Common Divisor}

Suppose we are given two polynomials in one variable and with complex coefficients, only known approximately. We denote the given polynomials by $a(x)$ and $b(x)$, and $d(x)=\operatorname{GCD}(a(x), b(x))$, with $\operatorname{deg}(d(x))=r$.

If we apply the algorithm taught in elementary school to compute the greatest common divisor of two natural numbers, then we repeatedly have to divide polynomials. This repetitive division is numerically unstable as it involves the subtraction of polynomials, which may lead to a dramatic loss of significant digits when the polynomials have coefficients of equal magnitude. Our approach to computing the greatest common divisor of polynomials with approximate coefficients hinges on two operations: root finding and interpolation. Any introductory course in numerical analysis describes numerically stable algorithms for these two operations.

For a given tolerance $\epsilon>0$, we define the numerical greatest common divisor of $a(x)$ and $b(x)$ as the monic polynomial whose roots are common to $a(x)$ and $b(x)$ within the given tolerance $\epsilon$. More precisely, if $a\left(\alpha_{i}\right)=0$, for $i=1,2, \ldots, \operatorname{deg}(a(x))$ and $b\left(\beta_{i}\right)=0$, for $i=1,2, \ldots, \operatorname{deg}(b(x))$, then we can rearrange the indices of the roots of the two polynomials so that the $r$ common roots appear with lowest indices. Then we write

$$
a(x)=\prod_{i=1}^{r}\left(x-\alpha_{i}\right) \prod_{i=r+1}^{\operatorname{deg}(a(x))}\left(x-\alpha_{i}\right)=d_{1}(x) \prod_{i=r+1}^{\operatorname{deg}(a(x))}\left(x-\alpha_{i}\right)
$$

and

$$
b(x)=\prod_{i=1}^{r}\left(x-\beta_{i}\right) \prod_{i=r+1}^{\operatorname{deg}(b(x))}\left(x-\beta_{i}\right)=d_{2}(x) \prod_{i=r+1}^{\operatorname{deg}(b(x))}\left(x-\beta_{i}\right)
$$

where $\left|\alpha_{i}-\beta_{i}\right| \leq \epsilon$, for $i=1,2, \ldots, r$, and $\left|\alpha_{i}-\beta_{j}\right|>\epsilon$, for all $i$ and $j$ with index higher than $r$. The polynomials $d_{1}(x)$ and $d_{2}(x)$ are numerical approximations for the greatest common divisor $d(x)$ of $a(x)$ and $b(x)$.

Now that we have numerical approximations for $d(x)$, we want to find $k(x)$ and $l(x)$ defined in (10). We determine $k(x)$ by interpolation at those roots of $b(x)$ not shared by $a(x)$ replacing in (10) $x$ by $\beta_{i}$, for $i=r+1, \ldots, \operatorname{deg}(b(x))$ :

$$
d\left(\beta_{i}\right)=k\left(\beta_{i}\right) a\left(\beta_{i}\right) \quad \text { or } \quad k\left(\beta_{i}\right)=\frac{d\left(\beta_{i}\right)}{a\left(\beta_{i}\right)}, \quad \text { for } i>r .
$$

Note that as $i>r: a\left(\beta_{i}\right) \neq 0$. The interpolation conditions in (15) determine $k(x)$ uniquely as a polynomial of degree $\operatorname{deg}(b(x))-r-1$. Analogously, we determine $l(x)$ by interpolation at those roots of $a(x)$ not shared by $b(x)$ replacing in the equality (10) $x$ by $\alpha_{i}$, for $i=r+1, \ldots, \operatorname{deg}(a(x))$ :

$$
d\left(\alpha_{i}\right)=l\left(\alpha_{i}\right) b\left(\alpha_{i}\right) \quad \text { or } \quad l\left(\alpha_{i}\right)=\frac{d\left(\alpha_{i}\right)}{b\left(\alpha_{i}\right)}, \quad \text { for } i>r .
$$

Note that as $i>r: b\left(\alpha_{i}\right) \neq 0$. The interpolation conditions in (16) determine $l(x)$ uniquely as a polynomial of degree $\operatorname{deg}(a(x))-r-1$. 


\subsection{Numerical Experiments}

The algorithms described above have been implemented in C. In this section we list some numerical results, obtained by practical comparisons between our new algorithm and the elementary approach, for random and specific input data.

We call the elementary school algorithm by repetitive division the "naive algorithm" and the algorithm with root finding the "advanced algorithm". When the tolerance of $10^{-8}$ is used to decide whether two numbers are equal, the naive algorithm runs much faster than the other one, while the advanced algorithm is more numerically stable when the polynomial degree is less than 30 and the degree of the GCD is less than 15. Although the advanced algorithm is relatively slow, the time spent for each GCD computation is just trivial, less than 10 milliseconds even for the polynomial of degree 30 and the GCD of degree 15 . Table 2 summarizes this experiment.

\begin{tabular}{|c|c|c|c|}
\hline degree of $a$ and $b$ & degree of GCD & naive(\%) & advanced(\%) \\
\hline \hline 5 & 3 & 100.0 & 100.0 \\
10 & 5 & 99.2 & 100.0 \\
15 & 8 & 99.5 & 100.0 \\
20 & 10 & 99.5 & 100.0 \\
25 & 13 & 98.2 & 100.0 \\
30 & 15 & 88.2 & 100.0 \\
\hline
\end{tabular}

Table 2: Comparison of the naive algorithm and the advanced algorithm on random data. The first column lists the degree of the given polynomials $a$ and $b$. The second column lists the degree of the GCD. The data in columns three and four shows the percentage of success for 1000 tests of the two algorithms.

We also compared the performance of the algorithms for some specific data.

In the first experiment, the leading coefficient of the polynomial $b$ is $10^{-5}$ times leading coefficient of the polynomial $a$. We found that the naive algorithm fails completely when the degree $e$ of the input polynomials and the degree $r$ of the GCD satisfy the following relation:

$$
e= \begin{cases}2 r-1 & \text { for } r \text { is odd } \\ 2 r & \text { for } r \text { is even }\end{cases}
$$

Other relations between the degrees work fine. While the advanced algorithm remains numerically stable no matter what the relations between the degrees are. See the column with header specific numbers(1) in Table 3 for the experiment of this case.

Second, we test with some other specific numbers, say, if the higher degree coefficients of two polynomials are very near each other, then the two algorithms perform totally different. In particular, we tested polynomials with equal leading coefficients and with difference between the second-highest coefficient equal 
to $10^{-5}$. When the degree of the input polynomials is smaller than 5 and the degree of the GCD is smaller than 3, both algorithms work perfectly. When the degree of the input polynomials is larger than 10 and the degree of GCD is larger than 5, the naive algorithm fails completely, but the advanced algorithm shows the same numerical stability as on the random number case. See columns with header specific numbers(2) in Table 3 for the experiment of this case.

\begin{tabular}{|c|c|c|c|c|c|}
\hline \multicolumn{2}{|c|}{} & \multicolumn{2}{|c|}{ specific numbers(1) } & \multicolumn{2}{c|}{ specific numbers(2) } \\
\hline $\begin{array}{c}\text { degree } \\
\text { of } a \text { and } b\end{array}$ & $\begin{array}{c}\text { degree } \\
\text { of GCD }\end{array}$ & $\begin{array}{c}\text { naive } \\
\operatorname{method}(\%)\end{array}$ & $\begin{array}{c}\text { advanced } \\
\operatorname{method}(\%)\end{array}$ & $\begin{array}{c}\text { naive } \\
\operatorname{method}(\%)\end{array}$ & $\begin{array}{c}\text { advanced } \\
\operatorname{method}(\%)\end{array}$ \\
\hline \hline 5 & 3 & 0.0 & 100.0 & 99.9 & 100.0 \\
10 & 5 & 99.1 & 100.0 & 0.1 & 100.0 \\
15 & 8 & 98.8 & 100.0 & 0.0 & 100.0 \\
20 & 10 & 0.3 & 100.0 & 0.0 & 100.0 \\
\hline
\end{tabular}

Table 3: Comparison of the naive algorithm and the advanced algorithm on specific data. The first column lists the degree of the given polynomials $a$ and $b$. The second column contains the degree of the GCD. The data in columns three to six shows the percentage of success for 1000 tests of the two algorithms.

These experiments provide us with practical evidence that for both random and specific inputs, the advanced algorithm shows its strong numerical stability. Concerning the speed of the GCD algorithm, the time needed of the realization algorithm is negligible compared to the calculation time of the feedback laws with homotopies.

\section{Realization of Multi-Input Multi-Output Sys- tems}

In the previous section, we gave the derivation of the transfer function of the dynamic compensator as the output of the homotopies. We will give a modified algorithm based on [1, pages 389-416] to obtain minimal or irreducible realizations, which realize a system with the least number of dynamic elements. These modifications were made to fit the output format of the software used to compute the feedback laws. The necessity of the modifications will be discussed at the end of this section. We will show how to obtain realizations $\left\{F_{c}, G_{c}, H_{c}, K_{c}\right\}$ of the transfer function $T(s)$ in controller form first. Then, we will use the property of the output of homotopies to show the realizations are irreducible, so they are also controllable.

From (6), the transfer function can be written as

$$
T(s)=V(s) U^{-1}(s) .
$$

In accordance with convention, we would replace $V(s)$ by $N(s)$ which stands 
for numerator, replace $U(s)$ by $D(s)$ which stands for denominator.

According to Theorem 3.3 in [1 page 391], realizations exist if and only if $T(s)$ is a matrix of rational functions and satisfies

$$
\lim _{s \rightarrow \infty} T(s)<\infty,
$$

i.e., if and only if $T(s)$ is a proper rational matrix. Given the transfer function matrix $T(s)=N(s) D^{-1}(s)$ as a $(m \times p)$ proper rational matrix. Let $d_{j}=$ the highest degree of $j$ th column in the $D(s)\left(d_{j} \geq 0, j=1,2, \ldots, p\right)$. Define

$$
\Lambda(s)=\operatorname{diag}\left(s^{d_{1}}, \ldots, s^{d_{p}}\right),
$$

and

$$
S(s)=\text { block diag }\left(\left[\begin{array}{c}
1 \\
s \\
\vdots \\
s^{d_{j}-1}
\end{array}\right] j=1, \ldots, p\right) .
$$

If $d_{j}=0$, just skip that column and continue to fill the next column of the $S(s)$ matrix. Note that $S(s)$ is an $q\left(=\sum_{j=1}^{p} d_{j}\right) \times p$ polynomial matrix. Write

$$
D(s)=D_{h} \Lambda(s)+D_{l} S(s)
$$

$D_{h}$ is the highest column degree coefficient matrix of $D(s)$. For example, if $D(s)=\left[\begin{array}{cc}3 s^{2}+1 & 2 s \\ 2 s & s\end{array}\right]$, then the highest column degree coefficient matrix $D_{h}=\left[\begin{array}{ll}3 & 2 \\ 0 & 1\end{array}\right]$, and $D_{l} S(s)$ given in (22) accounts for the remaining lower column degree terms $D(s)$, with $D_{l}$ being a matrix of coefficients.

In general, $\left|D_{h}\right| \neq 0$, and define $p \times p$ and $p \times q$ matrices

$$
G_{p}=D_{h}^{-1}, \quad F_{p}=-D_{h}^{-1} D_{l},
$$

respectively. Then $F_{c}, G_{c}$ can be determined from

$$
F_{c}=\bar{F}_{c}+\bar{G}_{c} F_{p}, \quad G_{c}=\bar{G}_{c} G_{p},
$$

where $\bar{F}_{c}=\operatorname{block} \operatorname{diag}\left[F_{1}, F_{2} \ldots, F_{p}\right]$ with

$$
\begin{aligned}
& F_{j}=\left[\begin{array}{cccc}
0 & & \\
\vdots & & I_{d_{j-1}} & \\
0 & 0 & \cdots & 0
\end{array}\right] \in R^{d_{j} \times d_{j}}, \\
& \bar{G}_{c}=\text { block diag }\left(\left[\begin{array}{c}
0 \\
\vdots \\
0 \\
1
\end{array}\right] \in R^{d_{j}}, j=1, \ldots, p\right) \text {. }
\end{aligned}
$$


When $d_{j}=0$, we just skip the corresponding $F_{j}$ and continue to fill the $\bar{F}_{c}$ matrix with the $F_{j+1}$ matrix; we also need to add a zero column at the $j$ th column of the $\bar{G}_{c}$ matrix.

Then we can determine $H_{c}$ and $K_{c}$ such that

$$
N(s)=H_{c} S(s)+K_{c} D(s),
$$

and note that

$$
K_{c}=\lim _{s \rightarrow \infty} T(s) .
$$

Therefore, $H_{c}$ can be determined from (27).

An $q$ th-order realization of $T(s)$ in controller form is now given by the equations

$$
\dot{\mathbf{z}}_{c}=F_{c} \mathbf{z}_{c}+G_{c} \mathbf{y}, \quad \mathbf{u}=H_{c} \mathbf{z}_{c}+K_{c} \mathbf{y} .
$$

According to the format of the output of the software, $q=\sum_{j=1}^{p} d_{j}$ is equal to the minimal order of the dynamic compensator. Therefore, this algorithm gives us a minimal realization of the transfer function matrix $T(s)$ and the result is also observable.

The main difference between the modified algorithm and the original algorithm given in 1 is that the original algorithm limits $d_{j} \geq 1$, while our modified algorithm works for $d_{j} \geq 0$, where $d_{j}$ is the highest column degree of $j$ th column in the $D(s)$. Some $d_{j}$ must be equal to zero when the number of output is larger than the order of the dynamic compensator. In this case the modified algorithm become necessary. The correctness of the modified algorithm is verified with experiments.

\section{Software}

The dynamic feedback laws were calculated with the aid of PHCpack 34. While the second public release of PHCpack implemented the dynamic pole placement problem in its geometric form, additional software had to be written, concerning:

0. a limit on the number of feedback laws;

1. an interface between Ada and C; and

2. a collection of $\mathrm{C}$ routines for the realization.

The limit on the number of feedback laws was imposed as a matter of convenience, to control the practical complexity. We elaborate the other two items in the following subsections. 


\subsection{A C interface to PHCpack}

PHCpack is written in Ada, while the programs to process the feedback laws are in the lower level language $\mathrm{C}$.

We can build a portable interface to the Ada routines in PHCpack with $\mathrm{C}$ functions because the language Ada has the pragma Import construction to call routines from other languages such as $\mathrm{C}$ and it supports conversions for $\mathrm{C}$ integers, doubles, and arrays of these $\mathrm{C}$ types. Furthermore, the gnu-ada compiler provides a mechanism to call Ada routines from a $\mathrm{C}$ main program and to call $\mathrm{C}$ functions from Ada. As the gnu-ada compiler is integrated in the gcc compilation system, our interface is portable. In particular, we ran our implementation successfully on SUN workstations running Solaris and on PCs running Linux and Windows.

To exchange data efficiently, programs in Ada or C should define exchange protocols of structured data types into basic data types for which automatic conversions are supported. More precisely, we represent structured data types into arrays of doubles and arrays of integers. The language $\mathrm{C}$ is restricted in returning dynamically allocated variables. Therefore, data allocated in a $\mathrm{C}$ function is passed by the $\mathrm{C}$ function calling an Ada function for further processing of the data.

A typical sequence of calls goes as follows. First a $\mathrm{C}$ function gathers problem data and prepares the input to an Ada routine of PHCpack. The Ada routine, called from $\mathrm{C}$, uses path tracking to solve the problem, and then calls a $\mathrm{C}$ function to process the results obtained with PHCpack. So the $\mathrm{C}$ programmer who uses PHCpack should thus provide two C functions: one to prepare the input and one to process the output. This "hand-in-glove" interface is appropriate for a $\mathrm{C}$ programmer collaborating with an Ada programmer (which is the case of the authors), who only have to agree on the prototypes of the routines.

\subsection{The Organization of the Software}

In Figure 3 the arrows indicate the order of function calls in the computation and realization of the dynamic feedback laws. In this section we give a short description for each of the procedures, some written in C, others in Ada.

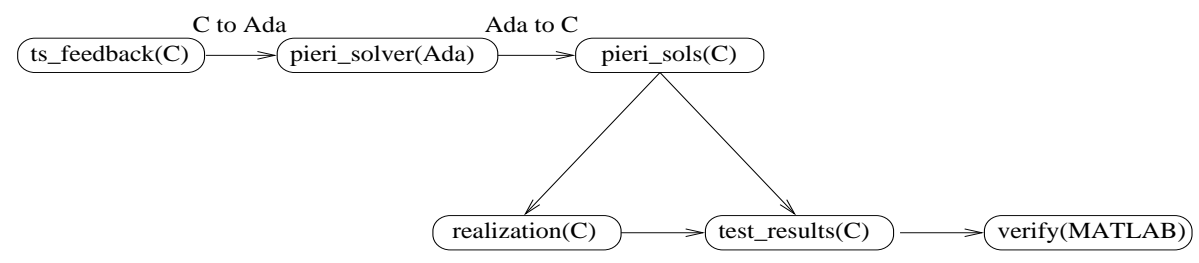

Figure 3: Organization of the Software

ts_feedback(C): ts_feedback reads all the input information from a file, includ- 
ing the number of the internal states $n$, the input dimension $m$, the output dimension $p$, the number of the internal states for the dynamic compensator $q$ and the number of output feedback. Also the user should give the $A, B, C$ matrices (or let the matrices be generated randomly) of the given plant and $n+q$ eigenvalues. ts_feedback.c computes $C\left(s I_{n}-A\right)^{-1} B$ at the interpolation points as the input planes. With $\mathrm{C}$ to Ada interface, we pass the arrays of the input planes and the interpolation points to the pieri_solver (an Ada procedure in PHCpack).

pieri_solver(Ada): The pieri_solver calculates the corresponding dynamic output feedbacks and passes them to the $\mathrm{C}$ program pieri_sols.c.

pieri_sols(C): With Ada to $\mathrm{C}$ interface, the arrays in Ada form are converted to the form in C. Then pieri_sols.c calls realization.c and tests the results.

realization(C): We use modified realization algorithm based on [1] to get the realization of the dynamic output feedback, organized as follows:

(a) Get the transfer function $T(s)=N(s) D(s)^{-1}$ from the output of the Ada program. The inverse of a polynomial matrix is a rational polynomial matrix and it is mainly done by Poly_Smith. See (10) in section 3 for more detail about how Smith form can work for the inverse of a polynomial matrix.

(b) The realization function implements the modified realization algorithm to get a minimal realization of the dynamic compensator.

(c) Evaluate the transfer function $T(s)$ at some random point and compare it with the result after realization $\left(H\left(s I_{q}-F\right)^{-1} G+K\right)$ at the same point. If the values are the same, the realization is correct.

test_results(C): We can evaluate equation (2) at the given poles and calculate the determinant with the previous result. If the determinant is zero, the pole is the eigenvalue of the closed-loop system. As mentioned above, equation (2) is algebraically equal to equation (11), which is the characteristic equation of the closed-loop system.

verify(MATLAB): Finally, a MATLAB script verifies the results by comparing the computed poles with the given poles and finding the condition number for each given pole.

\subsection{Availability of the Software}

The $\mathrm{C}$ routines for the numerical realization algorithms written by Yusong Wang are available at http://www.math.uic.edu/ jan in the distribution of release 2.2 of the source code of PHCpack (see [34], the first version of this package is archived by Netlib). In particular, the collection of routines can be found in the directory "Feedback" of the source code. Also the input data files for the applications discussed in the next section are available in this directory. 


\section{Applications}

In this section we illustrate the usefulness of our approach. Some application examples will show it is necessary to find some dynamic output feedback laws for some specific situations, especially for the cases there is no feedback laws or no real feedback laws at some given poles for the static feedback problem.

We assume that the input data for our applications is given as $(A, B, C)$, i.e.: a triple of three matrices of the linear system $\dot{\mathbf{x}}=A \mathbf{x}+B \mathbf{u}$, and $\mathbf{y}=C \mathbf{x}$, where $\mathbf{x}, \mathbf{u}$, and $\mathbf{y}$ are vectors of internal states, input, and output respectively.

\subsection{Satellite Trajectory Control}

This application concerns the design of output feedback laws to keep a satellite in orbit. We treated this problem in our previous paper [35] with static output feedback and with ad hoc MATLAB scripts to aid the manipulations. In [35], we already found two real output feedback laws for static case. Now we want to get some feedback laws for dynamic case as an extension of our method.

The linearized state-space equations for the satellite problem are given below:

$$
\begin{aligned}
& A=\left[\begin{array}{cccc}
0 & 1 & 0 & 0 \\
0.3578 & 0 & 0 & 0.8525 \\
0 & 0 & 0 & 1 \\
0 & -0.5596 & 0 & 0
\end{array}\right] ; \\
& B=\left[\begin{array}{cc}
0 & 0 \\
1.3411 & 0 \\
0 & 0 \\
0 & 1.0867
\end{array}\right] .
\end{aligned}
$$

We choose value for each parameter randomly. While this may seem unrealistic, our choice can be justified by an appropriate selection of units. More detail about the model can be found in [7, 16]. We define $C$ as some random matrix - which can be interpreted as a random projection of the states onto a plane.

For this satellite example, we have $n=4, m=p=2$, which is dimension zero for the static case. When $q=1$, the system become underdetermined since $n+q=5<m p+q(m+p)=8$ and there are three degrees of freedom. We choose the eigenvalues as $\left(\frac{-2+i}{\sqrt{5}}, \frac{-2-i}{\sqrt{5}},-5,-7,-3.0,-0.1068,-0.7834,-0.9582\right)$. The last three of eigenvalues are randomly selected. We found two real feedback laws and six complex complex feedback laws. We also use the MATLAB script to verify the results and calculate the condition number for the eigenvalue $\lambda_{i}$ by

$$
\frac{1}{\left|\mathbf{y}_{i}^{H} \mathbf{x}_{i}\right|}, \quad i=1,2, \ldots, n+q,
$$

where vectors $\mathbf{x}_{i}$ and $\mathbf{y}_{i}$ denote the unit right and left eigenvectors of the closedloop system. See [1] page 323] for the derivation of $\left|\mathbf{y}_{i}^{H} \mathbf{x}_{i}\right|$ as the reciprocal of 
the condition number for the eigenvalue $\lambda_{i}$. By substituting the result into the closed-loop system, we find the relative difference of the computed eigenvalues vs. the given eigenvalues is bounded by $10^{-11}$ and the order of condition numbers are at most $10^{3}$, calculated with (31). The total CPU time spent on the calculation of the dynamic feedback laws is 2 seconds and 320 milliseconds on a $2.4 \mathrm{GHz}$ workstation running Linux System.

\subsection{Numerical Example}

In this section we report on two numerical examples in the literature [27] [36].

Numeric Example A: We will use the example in 27] to illustrate the following situation: when a system is overdetermined for static output feedback $(n>m p, q=0)$, for which no feedback laws can be found at the desired poles, we can choose a q to make it convert to underdetermined or dimension zero case $(n+q \leq m p+q(m+p))$, for which we can find feedback laws.

Consider the system, given by

$$
\begin{aligned}
A & =\left[\begin{array}{rrrrrr}
0 & 0 & 0 & 0 & 0 & 0 \\
1 & 0 & 0 & 0 & 0 & -1 \\
0 & 1 & 0 & 0 & 0 & 0 \\
0 & 0 & 0 & 0 & 0 & 0 \\
0 & 0 & 0 & 1 & 0 & 0 \\
0 & 0 & -1 & 0 & 1 & 0
\end{array}\right] ; \\
B & =\left[\begin{array}{rr}
1 & 3 \\
0 & 0 \\
0 & -1 \\
0 & 1 \\
0 & 1 \\
0 & 0
\end{array}\right] ; \quad C=\left[\begin{array}{llllll}
0 & 0 & 1 & 0 & 0 & 0 \\
0 & 0 & 0 & 0 & 0 & 1
\end{array}\right] .
\end{aligned}
$$

Here, $m=p=2, n=6$, therefore $n>m p$ which is overdetermined for static case. We will choose a $q$ to make the system underdetermined. The minimum possible $q$ is 1 , then we have $n+q=7<m p+q(m+p)=$ 8 , so there is one degree of freedom. With the calculation of PHCpack and the $\mathrm{C}$ interface, we can easily get some dynamic compensators of McMillan degree $q=1$ to control the system. We verified the result by comparing the given and computed eigenvalues with a MATLAB script. For a choice $(-0.1,-1.5,-0.9,-0.7,-6.0,-3.5,-8.0)$ of 7 eigenvalues and an additional pole -0.1053 which is generated by random, the relative difference of the computed eigenvalues vs. the given eigenvalues of the closed-loop system is bounded by $10^{-9}$. In this specific example, we find 8 solutions and 4 of them are real (sometimes 6 are real, depending on the

additional input plane which are randomly generated). The order of the 
condition number computed with (31) is no more than $10^{3}$ for all of the given eigenvalues. The total CPU time spent for this numeric example is around 3 seconds with the workstation mentioned above.

Numeric Example B: The second example can be found in [36. Example 3.7], with $n=8$ and $m=p=3$.

The system is given as below

$$
\begin{aligned}
A & =\left[\begin{array}{rrrrrrrr}
0 & -1 & 0 & 0 & 0 & 0 & 0 & 1 \\
1 & 2 & 0 & 0 & 1 & 0 & 0 & -2 \\
0 & -1 & 0 & 0 & 5 & 0 & 0 & 0 \\
0 & 0 & 1 & 0 & -7 & 0 & 0 & -2 \\
0 & -1 & 0 & 1 & 4 & 0 & 0 & 2 \\
0 & -2 & 0 & 0 & 2 & 0 & 0 & 3 \\
0 & 0 & 0 & 0 & -1 & 1 & 0 & -2 \\
0 & -1 & 0 & 0 & 1 & 0 & 1 & -1
\end{array}\right] \\
B & =\left[\begin{array}{rrr}
0 & 1 & 2 \\
1 & 0 & 1 \\
-1 & -1 & -3 \\
1 & 0 & 1 \\
0 & 2 & 4 \\
2 & 1 & 5 \\
-1 & 1 & 1 \\
1 & -1 & -1
\end{array}\right] ; \\
C & =\left[\begin{array}{rrrrrrrr}
0 & 1 & 0 & 0 & -2 & 0 & 0 & 0 \\
0 & 0 & 0 & 0 & 1 & 0 & 0 & 0 \\
0 & 0 & 0 & 0 & 0 & 0 & 0 & 1
\end{array}\right]
\end{aligned}
$$

This system is underdetermined for static output feedback $(n<m p, q=$ $0)$. When the given poles are $(-0.8090+0.5878 i,-0.9511-0.3090 i,-0.3090-$ $0.9511 i,-0.3090+0.9511 i,-0.9511+0.3090 i,-0.8090-0.5878 i,-0.5878+$ $0.8090 i,-0.5878-0.8090 i,-0.1883)$, in which the first 8 are picked at the unit circle and the last one is a negative real number chosen by random, we find 42 feedback laws with our software and 4 of them are real. The relative difference of the computed eigenvalues vs. the given eigenvalues is $10^{-9}$. The order of the condition number is bounded by $10^{5}$ if computed with (31). The total CPU time spent on this example is around 50 seconds to find all 42 feedback laws. It only takes 1 second and 180 milliseconds if user just needs one feedback law.

\subsection{Aircraft Control}

It may be that for a given selection of poles, all static feedback laws have coefficients with nonzero imaginary parts. In this case, we will design dynamic 
output feedback laws, and exploit the additional freedom to place the poles at their originally selected locations. The model from MathWorks 21] could be used to illustrate this kind of situation. It also can be found in any standard text in aviation for a more complete discussion of the physics behind aircraft flight. The state-space equations are given below.

The jet model during cruise flight at $\mathrm{MACH}=0.8$ and $\mathrm{H}=40,000 \mathrm{ft}$. is

$$
\begin{aligned}
& A=\left[\begin{array}{rrrr}
-0.0558 & -0.9968 & 0.0802 & 0.0415 \\
0.5980 & -0.1150 & -0.0318 & 0 \\
-3.0500 & 0.3880 & -0.4650 & 0 \\
0 & 0.0850 & 1.0000 & 0
\end{array}\right] ; \\
& B=\left[\begin{array}{cc}
0.0073 & 0 \\
-0.4750 & 0.0077 \\
0.1530 & 0.1430 \\
0 & 0
\end{array}\right] ; \quad C=\left[\begin{array}{llll}
0 & 1 & 0 & 0 \\
0 & 0 & 0 & 1
\end{array}\right] .
\end{aligned}
$$

For this example, $n=4, m=p=2$, so $n=m p$ when $q=0$, which is the dimension zero case. We can find some feedback laws with PHCpack. When the chosen poles are $(-0.234,-1+3.2 i,-1-3.2 i,-3.0)$, we found two complex feedback laws. The total time spent is 140 milliseconds and the realization part just needs trivial time. By computing the eigenvalues of the closed-loop system with the MATLAB script, we find the difference between the computed eigenvalues and the given eigenvalues is just $10^{-14}$ and the condition number is around 10. Although the result seems quite nice, we still want to find some real feedback laws. We could choose $q=1$ to find some dynamic feedback laws, therefore $n+q=5<m p+q(m+p)=8$, then we have three degrees of freedom. With the calculation of PHCpack and C interface, we get 8 solutions, including 2 or 4 , or 6 real solutions, depending on different additional eigenvalues. For a choice of $(-0.234,-1+3.2 i,-1-3.2 i,-3.0,-7.0)$, in which the first four are the same as in the static case, and three randomly generated poles are $(-0.944,-0.995,-0.904)$, the relative difference of the computed eigenvalues vs. the given eigenvalues is bounded by $10^{-10}$. The total CPU time is 2 seconds and 680 milliseconds. For this choice of poles, we found four real feedback laws. For most of the 8 solutions, the condition numbers of the closed-loop system are less than $10^{2}$, and few of the solutions have the condition number $10^{4}$, calculated with formula (31).

\section{Conclusions}

In this paper we showed the practical feasibility of computing dynamic feedback laws using numerical homotopy algorithms, and we applied our software to examples from the control literature. Our publicly available implementation of the numerical greatest common divisor and numerical Smith normal form may be of independent interest. 


\section{References}

[1] P.J. Antsaklis and A.N. Michel. Linear Systems. McGraw-Hill, 1997.

[2] B. Beckermann and G. Labahn. Numeric and Symbolic Computation of problems defined by Structured Linear Systems. Reliable Computing 6:365-390, 2000.

[3] R.W. Brockett and C.I. Byrnes. Multivariate Nyquist criteria, root loci, and pole placement: a geometric viewpoint. IEEE Trans. Automat. Contr., 26:271-284, 1981.

[4] C.I. Byrnes. Pole assignment by output feedback. In Three Decades of Mathematical Systems Theory, edited by H. Nijmacher and J.M. Schumacher, pages 13-78. Springer-Verlag, Berlin, 1989.

[5] E.K. Chu. Optimization and pole assignment in control system design. International Journal of Applied Mathematics and Computer Science 11(5):1035-1053, 2001.

[6] R.M. Corless, E. Kaltofen, and S.M. Watt. Hybrid methods. In Computer Algebra Handbook, edited by J. Grabmeier, E. Kaltofen, and V. Weispfenning, pages 112-125, Springer-Verlag, 2002.

[7] D.C. Dorf and R.H. Bishop. Modern Control Systems. Addison-Wesley, 1998.

[8] I.Z. Emiris, A. Galligo, and H. Lombardi. Certified approximate univariate GCDs. J. Pure Applied Algebra 117\&118: 229-251, 1997.

[9] A. Eremenko and A. Gabrielov. Counterexamples to pole placement by static output feedback. Linear Algebra and Appl. 351-352: 211-218, 2002.

[10] A. Eremenko and A. Gabrielov. Pole placement by static output feedback for generic linear systems. SIAM J. Control Optim. 41(1):303-313, 2002.

[11] G.H. Golub and C.F. Van Loan. Matrix Computations. Third Edition. The Johns Hopkins University Press, 1996.

[12] D. Henrion and M. Šebek. Reliable numerical methods for polynomial matrix triangulation. IEEE Trans. Automat. Contr. 44(3):497-508, 1999.

[13] M.A. Hitz, E. Kaltofen, and Y.N. Lakshman. Efficient algorithms for computing the nearest polynomial with a real root and related problems. In ISSAC 99 Proc. 1999 Internat. Symp. Symbolic Algebraic Comput., edited by S. Dooley, pages 205-212, ACM, New York, 1999.

[14] B. Huber, F. Sottile, and B. Sturmfels. Numerical Schubert calculus. J. Symbolic Computation 26(6):767-788, 1998. 
[15] B. Huber and J. Verschelde. Pieri homotopies for problems in enumerative geometry applied to pole placement in linear systems control. SIAM J. Control Optim. 38(4):1265-1287, 2000.

[16] T. Kailath. Linear Systems. Prentice-Hall, 1980.

[17] N. Karmarkar and Y.N. Lakshman. Approximate polynomial greatest common divisors and nearest singular polynomials. In ISSAC 96 Proc. 1996 Internat. Symp. Symbolic Algebraic Comput., edited by Y.N. Lakshman, pages 35-42, ACM, New York, 1996.

[18] M. Kim, J. Rosenthal, and X. Wang. Pole Placement and matrix extension problems: A common point of view. SIAM J. Control. Optim., to appear.

[19] T.Y. Li, T. Sauer, and J.A. Yorke. The cheater's homotopy: an efficient procedure for solving systems of polynomial equations. SIAM J. Numer. Anal. 26(5):1241-1251, 1989.

[20] T.Y. Li, X. Wang, and M. Wu. Numerical Schubert calculus by the Pieri homotopy algorithm. SIAM J. Numer. Anal. 20(2):578-600, 2002.

[21] The MathWorks. Control System Toolbox. For Use with MATLAB. Getting Started. Version 5. June 2001.

[22] A.P. Morgan and A.J. Sommese. Coefficient-parameter polynomial continuation. Appl. Math. Comput., 29(2):123-160, 1989. Errata: Appl. Math. Comput. 51:207(1992).

[23] V. Pan. Solving a polynomial equation: some history and recent progress. SIAM Review 39(2):187-220, 1997.

[24] V. Pan. Computation of approximate polynomial GCDs and an extension. Information and Computation 167:71-85, 2001.

[25] M.S. Ravi, J. Rosenthal, and X. Wang. Dynamic pole placement assignment and Schubert calculus. SIAM J. Control Optim. 34(3):813-832, 1996.

[26] M.S. Ravi, J. Rosenthal, and X. Wang. Degree of the generalized Plücker embedding of a quot scheme and quatum cohomology. Math. Ann., 311:11-26, 1998.

[27] J. Rosenthal and X.A. Wang. Output Feedback Pole Placement with Dynamic Compensators. IEEE Trans. Automat. Contr. 41(6):830-843, 1996.

[28] J. Rosenthal and J.C. Willems. Open problems in the area of pole placement. In Open Problems in Mathematical Systems and Control Theory, edited by V.D. Blondel, E.D. Sontag, M. Vidyasagar, and J.C. Willems, pages 181-191. Springer-Verlag, 1998. 
[29] A.J. Sommese and J. Verschelde. Numerical homotopies to compute generic points on positive dimensional algebraic sets. Journal of Complexity 16(3):572-602, 2000.

[30] A.J. Sommese, J. Verschelde and C.W. Wampler. Numerical decomposition of the solution sets of polynomial systems into irreducible components. SIAM J. Numer. Anal. 38(6):2022-2046, 2001.

[31] A.J. Sommese, J. Verschelde and C.W. Wampler. Symmetric functions applied to decomposing solution sets of polynomial systems. SIAM J. Numer. Anal. 40(6):2026-2046, 2002.

[32] A.J. Sommese and C.W. Wampler. Numerical algebraic geometry. In The Mathematics of Numerical Analysis, volume 32 of Lectures in Applied Mathematics, edited by J. Renegar, M. Shub, and S. Smale, pages 749763, 1996. Proceedings of the AMS-SIAM Summer Seminar in Applied Mathematics, Park City, Utah, July 17-August 11, 1995, Park City, Utah.

[33] F. Sottile and B. Sturmfels. A sagbi basis for the quantum Grassmannian. J. Pure and Appl. Algebra 158(2-3): 347-366, 2001.

[34] J. Verschelde. Algorithm 795: PHCpack: A general-purpose solver for polynomial systems by homotopy continuation. ACM Transactions on Mathematical Software 25(2):251-276, 1999. Software available at http://www.math.uic.edu/ jan

[35] J. Verschelde and Y. Wang. Numerical Homotopy Algorithms for Satellite Trajectory Control by Pole Placement. Proceedings of MTNS 2002, Mathematical Theory of Networks and Systems (CDROM), Notre Dame, August 12-16, 2002.

[36] X.A. Wang. Grassmannian, central projection, and output feedback pole assignment of linear systems. IEEE Trans. Automat. Contr. 41(6):786$794,1996$.

[37] L.H. Zhi and M.-T. Noda. Approximate GCD of Multivariate Polynomials. In Proceedings of the Asian Symposium on Computer Mathematics, pages 9-18, Chiangmai, Thailand, 2000.

[38] L.H. Zhi and W.D. Wu. Nearest singular polynomial. J. Symbolic Comput. 26(6):667-675, 1998. Special issue on Symbolic Numeric Algebra for Polynomials, edited by S.M. Watt and H.J. Stetter. 